\title{
TAIL BEHAVIOR OF THE QUEUE SIZE AND WAITING TIME IN A QUEUE WITH DISCRETE AUTOREGRESSIVE ARRIVALS
}

\author{
BARA KIM, ${ }^{*}$ Korea University \\ KHOSROW SOHRABY, ${ }^{* *}$ University of Missouri, Kansas City
}

\begin{abstract}
Autoregressive arrival models are described by a few parameters and provide a simple means to obtain analytical models for matching the first- and second-order statistics of measured data. We consider a discrete-time queueing system where the service time of a customer occupies one slot and the arrival process is governed by a discrete autoregressive process of order 1 (a DAR(1) process) which is characterized by an arbitrary stationary batch size distribution and a correlation coefficient. The tail behaviors of the queue length and the waiting time distributions are examined. In particular, it is shown that, unlike in the classical queueing models with Markovian arrival processes, the correlation in the DAR(1) model results in nongeometric tail behavior of the queue length (and the waiting time) if the stationary distribution of the DAR(1) process has infinite support. A complete characterization of the geometric tail behavior of the queue length (and the waiting time) is presented, showing the impact of the stationary distribution and the correlation coefficient when the stationary distribution of the DAR(1) process has finite support. It is also shown that the stationary distribution of the queue length (and the waiting time) has a tail of regular variation with index $-\beta-1$, by finding an explicit expression for the tail asymptotics when the stationary distribution of the DAR(1) process has a tail of regular variation with index $-\beta$.
\end{abstract}

Keywords: Queue; discrete autoregressive process; geometric tail distribution; regular variation

2000 Mathematics Subject Classification: Primary 60K25

Secondary 68M20

\section{Introduction}

In traffic modeling, it is crucial to concentrate on those traffic characteristics which can be estimated efficiently from the measured data, can be given a meaningful physical interpretation, and, finally, have a measurable and dominant impact on the performance of the network. We believe that the time series models with relatively few parameters are well suited for 'accurate and meaningful' modeling of various traffic sources in high-speed applications. From that point of view, the autoregressive model of order 1 (the AR(1) model) is a good candidate to model input traffic in telecommunications networks.

The traditional time series models are driven by 'white Gaussian noise' sequences, resulting in simple additive properties. The advantage of such models is that they are quite flexible in

Received 3 February 2005; revision received 27 May 2006.

* Postal address: Department of Mathematics and Telecommunication Mathematics Research Center, Korea University,

1 Anam-dong, Sungbuk-ku, Seoul, 136-701, Korea. Email address: bara@korea.ac.kr

** Postal address: School of Computing and Engineering, University of Missouri, Kansas City, MO 64110, USA.

Email address: sohrabyk@umkc.edu 
capturing different correlation structures. However, they allow the stationary distribution to be Gaussian only. Furthermore, such models are mainly applicable in simulation experiments, or to obtaining certain asymptotic results limited to a large number of multiplexed sources [1], [3].

In contrast, in the classical teletraffic theory, Markovian arrival processes are constructed from exponential distributions, e.g. Markov-modulated Poisson processes. Teletraffic analysis using such models is usually tractable, due to the memoryless property of the exponential distributions. However, these models might be 'over-parameterized' if the aim is to capture accurately both the distribution and the correlation structure of the measured data. Furthermore, the model identification and estimation become a major task within this traditional framework.

In the continuous-time case, the Poisson process is the simplest Markovian arrival process. This process has been generalized by Cox [6], Consul and Jain [5], and Drezner and Farnum [8]. Different correlation structures have been discussed, but the stationary distributions in the proposed models were restricted to specific classes.

The non-Gaussian time series models have been extensively covered in the open literature. In [7] and [20], the general framework for their statistical analysis was covered. In [13], [19], and [17], time series generated by different innovation processes were discussed.

Despite their simplicity and their success in modeling complex data, there have been very few exact analyses of queueing systems with time series models. In the continuous-time case, Finch [10] and Finch and Pearce [11] considered an MA/M/1 system, which has a moving average model for the interarrival process and an exponential server. In the cases of moving average models of orders 1 and 2 for the interarrival times, explicit expressions for the tail behavior of the queue length distribution were provided. The approach of Finch and Pearce can be extended to any finite-order moving average model, although the complexity of computations increases exponentially with the order of the moving average model. In [14], a discrete-time moving average model was analyzed. Moving average models of orders 1 and 2 were both considered. The general approach of Franken et al. [12] is in principle applicable to more general arrival processes, but their results are more geared toward limit theorems and conditions on the existence of the solution. Tin [22] and Szekli et al. [21] investigated Markov renewal arrival processes with general service time distributions.

In this paper, we concentrate on the discrete-time case. In particular, we study the discrete autoregressive process of order 1 (the DAR(1) process) source models where the service time of a customer occupies one slot. The DAR(1) model has been shown to be a good model for variable-bit-rate-coded teleconference traffic [9]. For a discrete-time single-server queue with DAR(1) inputs, Hwang and Sohraby [15] and Hwang et al. [16] derived the probability generating functions of the stationary queue size and the stationary waiting time, respectively. Kim et al. [18] obtained the stationary distributions of the queue size and the waiting time in transform-free form using an embedded Markov chain and the 'Bernoulli arrivals see time averages' property. For a discrete-time multiserver queue with DAR(1) inputs, Choi et al. [4] obtained the stationary distributions of the queue size and the waiting time using the matrixanalytic method.

In this paper, we consider a discrete-time single-server queue with DAR(1) inputs. The main contributions of the paper are as follows.

1. Using the theory of analytic functions and the probability generating functions of the stationary queue size and the waiting time, we present a complete characterization of the geometric tail behavior of the queue size and the waiting time when the stationary distribution of the DAR(1) input process has finite support (see Theorem 3). 
2. When the stationary distribution of the DAR(1) input process has infinite support, we find that the tail distributions of the stationary queue size and the waiting time do not have geometric bounds. In particular, they are not asymptotically geometric (see Theorem 4).

3. When the stationary distribution of the $\mathrm{DAR}(1)$ input process has a tail of regular variation, we find that the stationary distributions of the queue size and the waiting time also have tails of regular variation, by finding asymptotics for the tail distributions of the stationary queue size and waiting time (see Theorem 5).

\section{Model description}

Let $B(t), t=0,1, \ldots$, be independent, identically distributed random variables. We assume that $B(t)$ takes nonnegative integer values and write $b_{i}=\mathrm{P}\{B(t)=i\}, i=0,1, \ldots \operatorname{ADAR}(1)$ process $\{Y(t): t=0,1, \ldots\}$ is defined by the regression equation

$$
Y(0)=B(0), \quad Y(t)=(1-\alpha(t)) Y(t-1)+\alpha(t) B(t), \quad t=1,2, \ldots,
$$

where $\alpha(t), t=1,2, \ldots$, are independent, identically distributed Bernoulli random variables with $\mathrm{P}\{\alpha(t)=0\}=\delta$ and $\mathrm{P}\{\alpha(t)=1\}=1-\delta$ (for $0<\delta<1$ ), and $\{\alpha(t): t=1,2, \ldots\}$ is assumed to be independent of $\{B(t): t=0,1, \ldots\}$. The following are basic properties of a $\operatorname{DAR}(1)$ process (see, for example, [16]).

- $\{Y(t)\}$ is stationary.

- The distribution of $Y(t)$ is the same as the distribution of $B(t)$, i.e.

$$
\mathrm{P}\{Y(t)=i\}=b_{i}, \quad i=0,1, \ldots
$$

- The autocorrelation function for $\{Y(t): t=0,1, \ldots\}$ is given by

$$
r(t):=\frac{\operatorname{cov}(Y(0), Y(t))}{\operatorname{var}(Y(0))}=\delta^{t}, \quad t=0,1, \ldots
$$

We consider the DAR(1)/D/1 queue, where the time is divided into slots of equal size and one slot is needed to serve a packet. We assume that packet arrivals occur at the beginnings of slots and that departures occur at the ends of slots. In the DAR(1) process, $Y(t), t=0,1, \ldots$, represents the number of packets that arrive at the beginning of the $t$ th slot. The order of service is assumed to be based on the first-come-first-served policy. A packet among those that arrive at the beginning of a given slot can be served during that slot if there are no previously arrived packets at the arrival epoch. Packets that find that the system is not empty at the arrival epoch have to wait until the server becomes available. We assume that the stability condition $\rho:=\mathrm{E}[B(0)]<1$ holds.

\section{Preliminaries}

Let $N(t)$ be the number of packets in the system immediately before possible arrivals at the beginning of the $t$ th slot. Then $N(t)$ evolves according to the evolution equation

$$
N(t+1)=\max \{N(t)+Y(t)-1,0\}, \quad t=0,1, \ldots
$$

Let

$$
\pi_{n}:=\lim _{t \rightarrow \infty} \mathrm{P}\{N(t)=n\}, \quad n=0,1, \ldots
$$


be the limiting probabilities of $\{N(t): t=0,1, \ldots\}$. It is known [18] that the $\pi_{n}, n=0,1, \ldots$, are given by

$$
\pi_{0}=\frac{1-\rho}{b_{0}+\delta \bar{a}_{1}}, \quad \pi_{n+1}=\frac{1}{b_{0}+\delta \bar{a}_{1}} \sum_{i=0}^{n} \pi_{i}\left(\bar{a}_{n+1-i}-\delta \bar{a}_{n+2-i}\right), \quad n=0,1, \ldots,
$$

where

$$
\bar{a}_{i}:=\sum_{j=i}^{\infty} a_{j}:=\sum_{j=i}^{\infty} \mathrm{P}\{X(Y-1)=j\}, \quad i=0,1, \ldots,
$$

and $X$ and $Y$ are independent random variables whose distributions are given by $\mathrm{P}\{X=i\}=$ $(1-\delta) \delta^{i-1}, i=1,2, \ldots$, and $\mathrm{P}\{Y=i\}=b_{i}, i=0,1, \ldots$ By a standard manipulation of (1), we obtain the following theorem.

Theorem 1. The probability generating function, П $(z)$, of the stationary distribution $\left\{\pi_{i}: i=\right.$ $0,1, \ldots\}$ of the queue size $\{N(t)\}$ is given by

$$
\Pi(z)=\frac{(1-\rho)(z-1)}{(z-\delta)\left(\bar{a}_{1}-A^{+}(z)\right)+b_{0}(z-1)}, \quad|z| \leq 1, z \neq 1,
$$

where

$$
A^{+}(z):=\sum_{i=1}^{\infty} a_{i} z^{i}=\sum_{i=2}^{\infty} b_{i} \frac{(1-\delta) z^{i-1}}{1-\delta z^{i-1}}, \quad|z| \leq 1 .
$$

Remark 1. Let $\tilde{N}(t)$ be the number of packets in the system after possible arrivals at the beginning of the $t$ th slot. Denote by $\tilde{\pi}_{n}, n=0,1, \ldots$, the stationary probabilities of $\{\tilde{N}(t)\}$. From the relation $N(t+1)=\max \{\tilde{N}(t)-1,0\}$, we have

$$
\pi_{0}=\tilde{\pi}_{0}+\tilde{\pi}_{1}, \quad \pi_{n}=\tilde{\pi}_{n+1}, \quad n=1,2, \ldots
$$

We note that $\sum_{n=1}^{\infty} \tilde{\pi}_{n}$, which is the probability that the server is busy, is the mean number of customers in the service position. In addition, $\rho$ is the mean number of customers that arrive in any slot, and the sojourn time in the service position for a customer who enters the service position is one slot (length). Hence, by applying Little's formula (see, for example, [23]) to the service position, we have

$$
\sum_{n=1}^{\infty} \tilde{\pi}_{n}=\rho
$$

From (5) and (6), we obtain $\tilde{\pi}_{n}, n=0,1, \ldots$, as follows:

$$
\tilde{\pi}_{0}=1-\rho, \quad \tilde{\pi}_{1}=\pi_{0}-1+\rho, \quad \tilde{\pi}_{n}=\pi_{n-1}, \quad n=2,3, \ldots
$$

From (3) and (7), we find the probability generating function, $\tilde{\Pi}(z):=\sum_{n=0}^{\infty} \tilde{\pi}_{n} z^{n}$, of the distribution $\left\{\tilde{\pi}_{n}: n=0,1, \ldots\right\}$ :

$$
\begin{aligned}
\tilde{\Pi}(z) & =z \Pi(z)-(1-\rho)(z-1) \\
& =\frac{(1-\rho)(z-1)\left[z-b_{0}(z-1)-(z-\delta)\left(\bar{a}_{1}-A^{+}(z)\right)\right]}{(z-\delta)\left(\bar{a}_{1}-A^{+}(z)\right)+b_{0}(z-1)}, \quad|z| \leq 1, z \neq 1 .
\end{aligned}
$$


Since $\tilde{N}(t)$ evolves according to

$$
\tilde{N}(t+1)=\max \{\tilde{N}(t)-1,0\}+Y(t+1), \quad t=0,1, \ldots,
$$

the process $\{\tilde{N}(t)\}$ corresponds to the queue size process treated in [15]. Equation (8) is a reproduction of a result of [15].

Now we consider the stationary distribution of the waiting time. Let $w_{n}, n=0,1, \ldots$, denote the stationary probabilities of the waiting time.

Theorem 2. ([18].) We have the following relation between the stationary distribution of the queue size, $\left\{\pi_{n}: n=0,1, \ldots\right\}$, and the stationary distribution of the waiting time, $\left\{w_{n}: n=\right.$ $0,1, \ldots\}$ :

$$
\begin{aligned}
& w_{0}=\frac{1}{\rho}\left(a_{0}+(1-\rho) \bar{a}_{1}\right) \pi_{0}, \\
& w_{n}=\frac{1}{\rho} \pi_{n}, \quad n=1,2, \ldots
\end{aligned}
$$

\section{Geometric tails of the queue size and the waiting time}

Suppose that the stationary distribution, $\left\{b_{i}: i=0,1, \ldots\right\}$, of the DAR(1) inputs has finite support, i.e.

$$
i^{*}:=\sup \left\{i \geq 0: b_{i}>0\right\}<\infty .
$$

We assume that $i^{*} \geq 2$, to exclude the trivial cases. Let $f(z)$ denote the denominator of the right-hand side of (3), i.e.

$$
f(z):=(z-\delta)\left(\bar{a}_{1}-A^{+}(z)\right)+b_{0}(z-1), \quad|z| \leq 1 .
$$

Substituting (4) and $\bar{a}_{1}=\sum_{i=2}^{i^{*}} b_{i}$ into (10) leads to

$$
f(z)=(z-\delta) \sum_{i=2}^{i^{*}} b_{i} \frac{1-z^{i-1}}{1-\delta z^{i-1}}+b_{0}(z-1), \quad|z| \leq 1 .
$$

From (11), we observe that $f(z)$ has an analytic extension to $\left\{z \in \mathbb{C}:|z|<x^{*}\right\}$, where

$$
x^{*}:=\delta^{-1 /\left(i^{*}-1\right)} .
$$

We also denote the extended analytic function by $f(z),|z|<x^{*}$.

We have the following lemma.

Lemma 1. Suppose that $i^{*}:=\sup \left\{i \geq 0: b_{i}>0\right\}<\infty$.

(i) $f(z)$ has a unique zero on the interval $\left(1, x^{*}\right)$ of the real axis.

(ii) Let $\sigma$ be the zero of $f(z)$ on $\left(1, x^{*}\right)$. Then there is an $\varepsilon, 0<\varepsilon<x^{*}-\sigma$, such that $f(z)$ has no other zeros on $\{z \in \mathbb{C}:|z|<\sigma+\varepsilon\}$ except the two simple zeros 1 and $\sigma$.

Proof. (i) Define an analytic function $g(z)$ on $\left\{z \in \mathbb{C}:|z|<x^{*}\right\}$ by

$$
g(z)= \begin{cases}\frac{f(z)}{z-1}, & |z|<x^{*}, z \neq 1, \\ \lim _{z \rightarrow 1} \frac{f(z)}{z-1}, & z=1 .\end{cases}
$$


Since

$$
\bar{a}_{1}-A^{+}(z)=\sum_{i=1}^{\infty} a_{i}\left(1-z^{i}\right)=(1-z) \sum_{i=1}^{\infty} a_{i} \sum_{j=0}^{i-1} z^{j}=(1-z) \sum_{i=0}^{\infty} \bar{a}_{i+1} z^{i},
$$

(10) leads to

$$
g(z)=1-\sum_{i=0}^{\infty}\left(a_{i}+(1-\delta) \bar{a}_{i+1}\right) z^{i}, \quad|z|<x^{*} .
$$

By (12), $g(z)$ is a strictly decreasing function of $z$ on $\left(0, x^{*}\right)$. Let $X$ and $Y$ be independent random variables whose distributions are given by $\mathrm{P}\{X=i\}=(1-\delta) \delta^{i-1}, i=1,2, \ldots$, and $\mathrm{P}\{Y=i\}=b_{i}, i=0,1,2, \ldots$ Then

$$
\begin{aligned}
g(1) & =1-\bar{a}_{0}-(1-\delta) \sum_{i=1}^{\infty} \bar{a}_{i} \\
& =b_{0}-(1-\delta) \mathrm{E}\left[X(Y-1) \mathbf{1}_{\{Y>1\}}\right] \\
& =b_{0}-\mathrm{E}\left[(Y-1) \mathbf{1}_{\{Y>1\}}\right] \\
& =-\mathrm{E}[Y-1] \\
& =1-\rho \\
& >0 .
\end{aligned}
$$

By (11), we have

$$
\lim _{z \rightarrow x^{*}-} g(z)=-\infty
$$

The property that $g(z)$ is strictly decreasing on $\left(0, x^{*}\right)$, together with (13) and (14), implies that $g(z)$ has a unique zero on $\left(1, x^{*}\right)$. Thus, (i) is proved.

(ii) Let $\sigma$ be the zero of $f(z)$ and, hence, of $g(z)$ on $\left(1, x^{*}\right)$. By (12), for $|z|<\sigma$ we have

$$
|g(z)| \geq 1-\sum_{i=0}^{\infty}\left(a_{i}+(1-\delta) \bar{a}_{i+1}\right)|z|^{i}>1-\sum_{i=0}^{\infty}\left(a_{i}+(1-\delta) \bar{a}_{i+1}\right) \sigma^{i}=0 .
$$

Hence, $g(z)$ has no zeros on $\{z \in \mathbb{C}:|z|<\sigma\}$. Since $a_{i}+(1-\delta) \bar{a}_{i+1}>0, i=0,1, \ldots$, and $\sigma>0$ is a positive zero of $g(z)$, it can easily be shown from (12) that $g(z)$ has no zeros on $\{z \in \mathbb{C}:|z|=\sigma, z \neq \sigma\}$. Hence, there is an $\varepsilon, 0<\varepsilon<x^{*}-\sigma$, such that $g(z)$ has no zeros on $\{z \in \mathbb{C}:|z|<\sigma+\varepsilon, z \neq \sigma\}$. By (12), (d/dz)g(z)| $\left.\right|_{z=\sigma}<0$, which implies that the positive zero $\sigma$ of $g(z)$ is simple. Thus, (ii) is proved.

Theorem 3. Suppose that $i^{*}:=\sup \left\{i \geq 0: b_{i}>0\right\}<\infty$. Then the function $f(z)$ in (11) has a unique positive zero, $\sigma$, between 1 and $x^{*}:=\delta^{-1 /\left(i^{*}-1\right)}$.

(i) The stationary distribution, $\left\{\pi_{n}: n=0,1, \ldots\right\}$, of the queue size before possible arrivals, $\{N(t)\}$, has the following asymptotic tail behavior:

$$
\pi_{n}=c \sigma^{-n}+o\left(\sigma^{-n}\right) \text { as } n \rightarrow \infty .
$$

Here

$$
c=\frac{(1-\rho)(\sigma-1)(\sigma-\delta)}{\sigma\left[(\sigma-\delta)^{2}{A^{+}}^{\prime}(\sigma)-b_{0}(1-\delta)\right]}
$$

and ${A^{+}}^{\prime}(\sigma):=\left.(\mathrm{d} / \mathrm{d} z) A^{+}(z)\right|_{z=\sigma}$. 
(ii) The stationary distribution of the waiting time, $\left\{w_{n}: n=0,1, \ldots\right\}$, has the following asymptotic tail behavior, where $c$ is given by (16):

$$
w_{n}=\frac{c}{\rho} \sigma^{-n}+o\left(\sigma^{-n}\right) \text { as } n \rightarrow \infty .
$$

Proof. By Lemma 1(i), $f(z)$ has a unique positive zero, $\sigma$, between 1 and $x^{*}$. By Lemma 1(ii), the probability generating function $\Pi(z)$ in (3) can be decomposed as

$$
\Pi(z)=\frac{-\sigma c}{z-\sigma}+\Xi(z), \quad|z|<\sigma+\varepsilon, z \neq \sigma
$$

where $\Xi(z)$ is an analytic function on $\{z \in \mathbb{C}:|z|<\sigma+\varepsilon\}$. We obtain (15) by the Taylor series expansion of (18) around $z=0$. We obtain (17) from (15) and (9).

Remark 2. 1. From (7), the stationary distribution, $\left\{\tilde{\pi}_{n}: n=0,1, \ldots\right\}$, of the queue size after possible arrivals, $\{\tilde{N}(t)\}$, has the following asymptotic tail behavior, where $\tilde{c}=c \sigma$ :

$$
\tilde{\pi}_{n}=\tilde{c} \sigma^{-n}+o\left(\sigma^{-n}\right) \quad \text { as } n \rightarrow \infty .
$$

2. Hwang et al. [16] obtained an approximation for the decay rate of the virtual waiting time under the condition that $X(Y-1)$ has a finite moment generating function around the origin, where $X$ and $Y$ are independent random variables whose distributions are given by

$$
\mathrm{P}\{X=i\}=(1-\delta) \delta^{i-1}, \quad i=1, \ldots, \quad \mathrm{P}\{Y=i\}=b_{i}, \quad i=0,1, \ldots
$$

By careful observation, it can be shown that this condition of Hwang et al. is identical to the condition that the stationary distribution, $\left\{b_{i}: i=0,1, \ldots\right\}$, of the DAR(1) inputs have finite support. Since the virtual waiting time is the queue size before possible arrivals, $\{N(t)\}$, the decay rate of the virtual waiting time is $\sigma^{-1}$. Therefore, the result of Hwang et al. [16] is an approximation for $\sigma^{-1}$ under the condition that $\left\{b_{n}: n=0,1, \ldots\right\}$ have finite support.

Now we consider the case in which the stationary distribution of the DAR(1) inputs does not have finite support. The tail distribution of a random variable $Z$ taking nonnegative integer values is said to have a geometric bound if there exist positive numbers $c_{1}$ and $\alpha_{1}<1$ such that $\mathrm{P}\{Z \geq n\} \leq c_{1} \alpha_{1}^{n}, n=0,1, \ldots$. It can easily be shown that the tail distribution of $Z$ has a geometric bound if and only if there exist positive numbers $c_{2}$ and $\alpha_{2}<1$ such that $\mathrm{P}\{Z=n\} \leq c_{2} \alpha_{2}^{n}, n=0,1, \ldots$ The following theorem shows that the tail distributions of the stationary queue size and the stationary waiting time do not have geometric bounds unless $\left\{b_{i}: i=0,1, \ldots\right\}$ has finite support.

Theorem 4. Suppose that $i^{*}:=\sup \left\{i \geq 0: b_{i}>0\right\}=\infty$. Then the tail distributions of the stationary queue size and the stationary waiting time do not have geometric bounds. In particular, they are not asymptotically geometric.

Proof. Recall that the arrival process is represented by a DAR(1) process, $\{Y(t): t=$ $0,1, \ldots\}$, whose stationary distribution is $\left\{b_{i}: i=0,1, \ldots\right\}$. For a positive integer $M>1$, let

$$
Y^{(M)}(t)=\min \{Y(t), M\}
$$


Then $\left\{Y^{(M)}(t): t=0,1, \ldots\right\}$ is a DAR(1) process with the stationary distribution $\left\{b_{i}^{(M)}: i=\right.$ $0,1, \ldots\}$, where

$$
b_{i}^{(M)}:= \begin{cases}b_{i}, & \text { if } i<M, \\ \sum_{j=M}^{\infty} b_{j}, & \text { if } i=M, \\ 0, & \text { if } i>M .\end{cases}
$$

Consider the DAR(1)/D/1 queue in which arrivals occur according to the DAR(1) process $\left\{Y^{(M)}(t)\right\}$. Let $N^{(M)}(t)$ be the queue size at the beginning of the $t$ th slot immediately before possible arrivals, and let

$$
\pi_{n}^{(M)}:=\lim _{t \rightarrow \infty} \mathrm{P}\left\{N^{(M)}(t)=n\right\}, \quad n=0,1, \ldots
$$

By Theorem 3,

$$
\pi_{n}^{(M)}=c^{(M)}\left(\sigma^{(M)}\right)^{-n}+o\left(\left(\sigma^{(M)}\right)^{-n}\right) \quad \text { as } n \rightarrow \infty,
$$

for some $c^{(M)}>0$ and $\sigma^{(M)} \in\left(1, \delta^{-1 /(M-1)}\right)$. Hence,

$$
\sum_{i=n}^{\infty} \pi_{i}^{(M)}=\frac{c^{(M)} \sigma^{(M)}}{\sigma^{(M)}-1}\left(\sigma^{(M)}\right)^{-n}+o\left(\left(\sigma^{(M)}\right)^{-n}\right) \quad \text { as } n \rightarrow \infty .
$$

From the recursions

$$
\begin{aligned}
N(t+1) & =\max \{N(t)+Y(t)-1,0\}, & & t=0,1, \ldots, \\
N^{(M)}(t+1) & =\max \left\{N^{(M)}(t)+Y^{(M)}(t)-1,0\right\}, & & t=0,1, \ldots,
\end{aligned}
$$

we have $\mathrm{P}\{N(t) \geq n\} \geq \mathrm{P}\{\tilde{N}(t) \geq n\}, n=0,1, \ldots$, if $N(0)=\tilde{N}(0)$ in distribution. Letting $n \rightarrow \infty$, we have

$$
\sum_{i=n}^{\infty} \pi_{i} \geq \sum_{i=n}^{\infty} \pi_{i}^{(M)}, \quad n=0,1, \ldots
$$

From (19) and (20), there exists a $c_{1}^{(M)}>0$ such that

$$
\sum_{i=n}^{\infty} \pi_{i} \geq c_{1}^{(M)}\left(\sigma^{(M)}\right)^{-n}, \quad n=0,1, \ldots
$$

Since $\sigma^{(M)} \in\left(1, \delta^{-1 /(M-1)}\right)$, we have $\sigma^{(M)} \rightarrow 1$ as $M \rightarrow \infty$. Hence, (21) implies that there exist no $c_{1}>0$ and $\alpha_{1}, 0<\alpha_{1}<1$, such that

$$
\sum_{i=n}^{\infty} \pi_{i} \leq c_{1} \alpha_{1}^{n}, \quad n=0,1, \ldots
$$

Therefore, the tail distribution of the stationary queue size before possible arrivals cannot have a geometric bound. Because of (9), the tail distribution of the stationary waiting time also cannot have a geometric bound.

From Theorems 3 and 4, we have the following corollary. 
Corollary 1. The following statements are equivalent.

(a) The stationary distribution of the DAR(1) process has finite support.

(b) The stationary distribution of the queue size is asymptotically geometric.

(c) The tail distribution of the stationary queue size has a geometric bound.

(d) The stationary distribution of the waiting time is asymptotically geometric.

(e) The tail distribution of the stationary waiting time has a geometric bound.

\section{Regularly varying tails for the queue size and the waiting time}

In this section, we consider the DAR(1)/D/1 queue where the stationary distribution of the DAR(1) input process has a regularly varying tail. A random variable $Z$ is said to have a tail distribution of regular variation with index $-\gamma, \gamma>0$, if its tail distribution has the following asymptotic behavior:

$$
\mathrm{P}\{Z \geq x\} \sim x^{-\gamma} L(x) \text { as } x \rightarrow \infty .
$$

Here $L(\cdot)$ is a slowly varying function, i.e. $L(\cdot)$ is a Lebesgue-measurable function that is ultimately positive and such that $\lim _{x \rightarrow \infty} L(\lambda x) / L(x)=1$ for all $\lambda>0$.

The following result is the main theorem in this section.

Theorem 5. Suppose that the stationary distribution of the DAR(1) process has a tail of regular variation with index $-\beta-1, \beta>0$, i.e.

$$
\sum_{i=n}^{\infty} b_{i} \sim n^{-\beta-1} L(n) \text { as } n \rightarrow \infty,
$$

for some slowly varying function $L(\cdot)$. Then the stationary distributions of the queue size and the waiting time have tails of regular variation with index $-\beta$, as follows.

(i) The stationary distribution, $\left\{\pi_{n}: n=0,1, \ldots\right\}$, of the queue size before possible arrivals, $\{N(t)\}$, satisfies

$$
\sum_{k=n}^{\infty} \pi_{k} \sim \frac{d}{\beta} n^{-\beta} L(n) \text { as } n \rightarrow \infty,
$$

where

$$
d=\frac{1-\delta}{1-\rho} \sum_{k=1}^{\infty}(1-\delta) \delta^{k-1} k^{\beta+1} .
$$

Furthermore,

$$
\pi_{n} \sim d n^{-\beta-1} L(n) \text { as } n \rightarrow \infty .
$$

(ii) The stationary distribution, $\left\{w_{n}: n=0,1, \ldots\right\}$, of the waiting time satisfies

$$
\sum_{k=n}^{\infty} w_{k} \sim \frac{d}{\rho \beta} n^{-\beta} L(n) \text { as } n \rightarrow \infty,
$$

where d is given by (24). Furthermore,

$$
w_{n}=\frac{d}{\rho} n^{-\beta-1} L(n) \text { as } n \rightarrow \infty .
$$


To prove Theorem 5, we need a series of lemmas.

Lemma 2. Suppose that $L(\cdot)$ is a slowly varying function. Then there exist $C, D$, and $x_{0}$ such that

$$
L(x) \leq C \lambda^{D} L(\lambda x) \quad \text { if } \lambda \geq 1 \text { and } x \geq x_{0} .
$$

Proof. See Corollary 2.0.5 of [2].

Lemma 3. Let $Z_{1}$ and $Z_{2}$ be independent random variables. Suppose that $Z_{1}$ takes nonnegative integer values and that $\mathrm{E}\left[Z_{1}^{n}\right]<\infty$ for all $n>0$. If

$$
\mathrm{P}\left\{Z_{2} \geq x\right\} \sim x^{-\gamma} L(x) \text { as } x \rightarrow \infty,
$$

for some $\gamma>0$ and a slowly varying function $L(\cdot)$, then

$$
\mathrm{P}\left\{Z_{1} Z_{2} \geq x\right\} \sim x^{-\gamma} \mathrm{E}\left[Z_{1}^{\gamma}\right] L(x) \text { as } x \rightarrow \infty .
$$

Proof. Without loss of generality, we assume that

$$
\mathrm{P}\left\{Z_{2} \geq x\right\}=x^{-\gamma} L(x), \quad x>0 .
$$

By Fatou's lemma,

$$
\begin{aligned}
\liminf _{x \rightarrow \infty} \frac{\mathrm{P}\left\{Z_{1} Z_{2} \geq x\right\}}{x^{-\gamma} L(x)} & =\liminf _{x \rightarrow \infty} \frac{\sum_{k=1}^{\infty} \mathrm{P}\left\{Z_{1}=k\right\} \mathrm{P}\left\{Z_{2} \geq x / k\right\}}{x^{-\gamma} L(x)} \\
& =\liminf _{x \rightarrow \infty} \frac{\sum_{k=1}^{\infty} \mathrm{P}\left\{Z_{1}=k\right\} k^{\gamma} L(x / k)}{L(x)} \\
& \geq \mathrm{E}\left[Z_{1}^{\gamma}\right] .
\end{aligned}
$$

Choose an $\alpha$ such that $\alpha>\gamma$. For $x>0$, let

$$
K(x):=\inf \left\{n \geq 1: \mathrm{P}\left\{Z_{1} \geq n\right\} \leq x^{-\alpha}\right\} .
$$

Let $K_{1}$ be an arbitrary positive integer. Since

$$
\begin{aligned}
\mathrm{P}\left\{Z_{1} Z_{2} \geq x\right\}= & \sum_{k=1}^{\min \left\{K_{1}, K(x)\right\}} \mathrm{P}\left\{Z_{1}=k\right\} \mathrm{P}\left\{Z_{2} \geq \frac{x}{k}\right\} \\
& +\sum_{k=\min \left\{K_{1}, K(x)\right\}+1}^{K(x)} \mathrm{P}\left\{Z_{1}=k\right\} \mathrm{P}\left\{Z_{2} \geq \frac{x}{k}\right\} \\
& +\sum_{k=K(x)+1}^{\infty} \mathrm{P}\left\{Z_{1}=k\right\} \mathrm{P}\left\{Z_{2} \geq \frac{x}{k}\right\}
\end{aligned}
$$


we have

$$
\begin{aligned}
\limsup _{x \rightarrow \infty} & \frac{\mathrm{P}\left\{Z_{1} Z_{2} \geq x\right\}}{x^{-\gamma} L(x)} \\
\leq & \limsup _{x \rightarrow \infty} \sum_{k=1}^{K_{1}} \mathrm{P}\left\{Z_{1}=k\right\} k^{\gamma} \frac{L(x / k)}{L(x)}+\limsup _{x \rightarrow \infty} \sum_{k=K_{1}+1}^{K(x)} \mathrm{P}\left\{Z_{1}=k\right\} k^{\gamma} \frac{L(x / k)}{L(x)} \\
& +\limsup _{x \rightarrow \infty} \frac{\mathrm{P}\left\{Z_{1} \geq K(x)+1\right\}}{x^{-\gamma} L(x)} \\
= & \sum_{k=1}^{K_{1}} \mathrm{P}\left\{Z_{1}=k\right\} k^{\gamma}+\limsup _{x \rightarrow \infty} \sum_{k=K_{1}+1}^{K(x)} \mathrm{P}\left\{Z_{1}=k\right\} k^{\gamma} \frac{L(x / k)}{L(x)} \\
& +\limsup _{x \rightarrow \infty} \frac{\mathrm{P}\left\{Z_{1} \geq K(x)+1\right\}}{x^{-\gamma} L(x)} .
\end{aligned}
$$

By (26),

$$
\limsup _{x \rightarrow \infty} \frac{\mathrm{P}\left\{Z_{1} \geq K(x)+1\right\}}{x^{-\gamma} L(x)} \leq \limsup _{x \rightarrow \infty} \frac{x^{-\alpha}}{x^{-\gamma} L(x)}=0 .
$$

Hence, (27) leads to

$$
\limsup _{x \rightarrow \infty} \frac{\mathrm{P}\left\{Z_{1} Z_{2} \geq x\right\}}{x^{-\gamma} L(x)} \leq \mathrm{E}\left[Z_{1}^{\gamma}\right]+\limsup _{x \rightarrow \infty} \sum_{k=K_{1}+1}^{K(x)} \mathrm{P}\left\{Z_{1}=k\right\} k^{\gamma} \frac{L(x / k)}{L(x)} .
$$

According to Lemma 2, there exist $C, D$, and $x_{0}$ such that

$$
\frac{L(x / k)}{L(x)} \leq C k^{D} \quad \text { if } \frac{x}{k} \geq x_{0} \text {. }
$$

Hence, if $x / K(x) \geq x_{0}$ then

$$
\sum_{k=K_{1}+1}^{K(x)} \mathrm{P}\left\{Z_{1}=k\right\} k^{\gamma} \frac{L(x / k)}{L(x)} \leq C \sum_{k=K_{1}+1}^{K(x)} \mathrm{P}\left\{Z_{1}=k\right\} k^{\gamma+D} \leq C \sum_{k=K_{1}+1}^{\infty} \mathrm{P}\left\{Z_{1}=k\right\} k^{\gamma+D} .
$$

Therefore, if

$$
\liminf _{x \rightarrow \infty} \frac{x}{K(x)}>x_{0}
$$

then

$$
\limsup _{x \rightarrow \infty} \sum_{k=K_{1}+1}^{K(x)} \mathrm{P}\left\{Z_{1}=k\right\} k^{\gamma} \frac{L(x / k)}{L(x)} \leq C \sum_{k=K_{1}+1}^{\infty} \mathrm{P}\left\{Z_{1}=k\right\} k^{\gamma+D} .
$$

Hence, by (28),

$$
\limsup _{x \rightarrow \infty} \frac{\mathrm{P}\left\{Z_{1} Z_{2} \geq x\right\}}{x^{-\gamma} L(x)} \leq \mathrm{E}\left[Z_{1}^{\gamma}\right]+C \sum_{k=K_{1}+1}^{\infty} \mathrm{P}\left\{Z_{1}=k\right\} k^{\gamma+D}
$$

if (29) holds. Letting $K_{1} \rightarrow \infty$ leads to

$$
\limsup _{x \rightarrow \infty} \frac{\mathrm{P}\left\{Z_{1} Z_{2} \geq x\right\}}{x^{-\gamma} L(x)} \leq \mathrm{E}\left[Z_{1}^{\gamma}\right]
$$

if (29) holds. Therefore, it remains to prove (29). 
We prove (29) by showing that

$$
\lim _{x \rightarrow \infty} \frac{K(x)}{x}=0
$$

Since $\mathrm{E}\left[Z_{1}^{\alpha+1}\right]<\infty$, we have $\sum_{n=1}^{\infty} n^{\alpha} \mathrm{P}\left\{Z_{1} \geq n\right\}<\infty$, whence $\lim _{n \rightarrow \infty} \mathrm{P}\left\{Z_{1} \geq n\right\} / n^{-\alpha}=$ 0 . Therefore,

$$
\lim _{x \rightarrow \infty} \frac{\mathrm{P}\left\{Z_{1} \geq \varepsilon x\right\}}{x^{-\alpha}}=0 \quad \text { for any } \varepsilon>0 .
$$

Hence, there exists an $M(\varepsilon)$ such that $\mathrm{P}\left\{Z_{1} \geq \varepsilon x\right\} \leq x^{-\alpha}$ if $x \geq M(\varepsilon)$. So, if $x \geq M(\varepsilon)$ then $K(x) \leq \varepsilon x$, which implies that

$$
\limsup _{x \rightarrow \infty} \frac{K(x)}{x} \leq \varepsilon .
$$

Since $\varepsilon>0$ is arbitrary, (30) holds and the proof is complete.

Lemma 4. Suppose that (22) holds. Then the elements of the sequence $\left\{\bar{a}_{n}: n=0,1, \ldots\right\}$, which were defined in (2), have the following asymptotic behavior:

$$
\bar{a}_{n} \sim n^{-\beta-1} \sum_{k=1}^{\infty}(1-\delta) \delta^{k-1} k^{\beta+1} L(n) \quad \text { as } n \rightarrow \infty .
$$

Proof. By (2), $\bar{a}_{n}=\mathrm{P}\{X(Y-1) \geq n\}, n=0,1, \ldots$ By (22), $\mathrm{P}\{Y \geq n\} \sim n^{-\beta-1} L(n)$ as $n \rightarrow \infty$. The result thus follows from Lemma 3 .

For probability distributions $\left\{p_{n}: n=0,1, \ldots\right\}$ and $\left\{q_{n}: n=0,1, \ldots\right\}$ on the set of nonnegative integers, let $\left\{(p * q)_{n}: n=0,1, \ldots\right\}$ denote the convolution of the two distributions, i.e.

$$
(p * q)_{n}:=\sum_{k=0}^{n} p_{k} q_{n-k}, \quad n=0,1, \ldots
$$

Lemma 5. Suppose that $\left\{p_{n}: n=0,1, \ldots\right\}$ and $\left\{q_{n}: n=0,1, \ldots\right\}$ are probability distributions on the set of nonnegative integers, and that

$$
p_{n} \sim n^{-\gamma} c_{1} L(n) \text { as } n \rightarrow \infty, \quad q_{n} \sim n^{-\gamma} c_{2} L(n) \text { as } n \rightarrow \infty,
$$

for some $c_{1}>0, c_{2}>0$, and $\gamma>1$ and a slowly varying function $L(\cdot)$. Then

$$
(p * q)_{n} \sim n^{-\gamma}\left(c_{1}+c_{2}\right) L(n) \text { as } n \rightarrow \infty .
$$

Proof. By the uniform convergence theorem for slowly varying functions [2],

$$
\lim _{x \rightarrow \infty} \frac{L(\lambda x)}{L(x)}=1 \quad \text { uniformly in } \lambda \in\left[\frac{1}{2}, 1\right] .
$$

By (31) and (32),

$$
\begin{aligned}
\limsup _{n \rightarrow \infty} \sup _{0 \leq k \leq n / 2} \frac{q_{n-k}}{n^{-\gamma} L(n)} & =\limsup _{n \rightarrow \infty} \sup _{0 \leq k \leq n / 2} \frac{q_{n-k}}{(n-k)^{-\gamma} L(n-k)}\left(\frac{n}{n-k}\right)^{\gamma} \frac{L((1-k / n) n)}{L(n)} \\
& =c_{2} 2^{\gamma} .
\end{aligned}
$$


Hence, by the Lebesgue dominated convergence theorem, we have

$$
\lim _{n \rightarrow \infty} \sum_{k=0}^{\lfloor n / 2\rfloor} \frac{p_{k} q_{n-k}}{n^{-\gamma} L(n)}=c_{2}
$$

where $\lfloor n / 2\rfloor$ denotes the largest integer which is not greater than $n / 2$. By interchanging the roles of $p_{k}$ and $q_{k}$, we obtain

$$
\lim _{n \rightarrow \infty} \sum_{k=\lfloor n / 2\rfloor+1}^{n} \frac{p_{k} q_{n-k}}{n^{-\gamma} L(n)}=\lim _{n \rightarrow \infty} \sum_{k=0}^{\lfloor(n-1) / 2\rfloor} \frac{p_{n-k} q_{k}}{n^{-\gamma} L(n)}=c_{1},
$$

and the proof is complete.

Let $\left\{p_{n}: n=0,1, \ldots\right\}$ be a probability distribution on the set of nonnegative integers. For $k=0,1, \ldots$, let $\left\{p_{n}^{* k}: n=0,1, \ldots\right\}$ denote the $k$-fold convolution of the distribution $\left\{p_{n}: n=0,1, \ldots\right\}$, i.e.

$$
p_{n}^{* 0}= \begin{cases}1, & n=0 \\ 0, & n=1,2, \ldots\end{cases}
$$

and, for $k=1,2, \ldots$,

$$
p_{n}^{* k}=\sum_{i=0}^{n} p_{i}^{*(k-1)} p_{n-i}, \quad n=0,1, \ldots
$$

Lemma 6. Let $\left\{p_{n}: n=0,1, \ldots\right\}$ be a probability distribution on the set of nonnegative integers. Suppose that $p_{n}>0, n=0,1, \ldots$, and that

$$
p_{n} \sim n^{-\gamma} L(n) \text { as } n \rightarrow \infty
$$

for some $\gamma>0$ and a slowly varying function $L(\cdot)$. Then, for any $\varepsilon>0$, there exists $a C(\varepsilon)>0$ such that

$$
p_{n}^{* k} \leq C(\varepsilon)(1+\varepsilon)^{k} p_{n}, \quad n, k=0,1, \ldots
$$

Proof. Let $\varepsilon>0$ be given. Choose a positive integer $m$ such that $(1+\varepsilon)^{m}>2$. By Lemma $5, p_{n}^{*(2 m)} \sim 2 p_{n}^{* m}$ as $n \rightarrow \infty$. Hence, there exists an $M>0$ such that

$$
p_{n}^{*(2 m)} \leq(1+\varepsilon)^{m} p_{n}^{* m} \text { for any } n \geq M .
$$

Let $C_{1}=\left(\min \left\{p_{0}^{* m}, \ldots, p_{M-1}^{* m}\right\}\right)^{-1}$.

We will show that

$$
p_{n}^{*(i m)} \leq C_{1}(1+\varepsilon)^{i m} p_{n}^{* m}, \quad n=0,1, \ldots,
$$

for $i=0,1, \ldots$, using induction on $i$. Trivially, (35) holds for $i \leq 1$. Suppose that (35) holds for $i=k$ with $k \geq 1$. By the induction hypothesis,

$$
p_{n}^{*(k+1) m}=\left(p^{*(k m)} * p^{* m}\right)_{n} \leq C_{1}(1+\varepsilon)^{k m} p_{n}^{* 2 m}, \quad n=0,1, \ldots
$$

Hence, by (34), $p_{n}^{*(k+1) m} \leq C_{1}(1+\varepsilon)^{(k+1) m} p_{n}^{* m}, n=M, M+1, \ldots$ Trivially, $p_{n}^{*(k+1) m} \leq$ $1 \leq C_{1} p_{n}^{* m} \leq C_{1}(1+\varepsilon)^{(k+1) m} p_{n}^{* m}, n=0, \ldots, M-1$. Thus, (35) holds for $i=k+1$ and, therefore, for all $i=0,1, \ldots$. 
By Lemma $5, p_{n}^{*(m+j)} \sim(m+j) p_{n}$ as $n \rightarrow \infty$, for $j=0, \ldots, m-1$. Hence, there exists a $C_{2}$ such that

$$
p_{n}^{*(m+j)} \leq C_{2} p_{n} \quad \text { for } j=0, \ldots, m-1 \text { and } n=0,1, \ldots
$$

By (35) and (36), for $i=0,1, \ldots$ and $j=0, \ldots, m-1$ we have

$$
p_{n}^{*(i m+j)} \leq C_{1} C_{2}(1+\varepsilon)^{i m} p_{n}, \quad n=0,1, \ldots
$$

Hence, (33) holds with $C(\varepsilon)=C_{1} C_{2}$.

Lemma 7. Let $\left\{p_{n}: n=0,1, \ldots\right\}$ and $\left\{q_{n}: n=0,1, \ldots\right\}$ be probability distributions on the set of nonnegative integers. Suppose that $\sum_{n=0}^{\infty} p_{n}(1+\varepsilon)^{n}<\infty$ for some $\varepsilon>0$, that $q_{n}>0$ for $n=0,1, \ldots$, and that $q_{n} \sim n^{-\gamma} L(n)$ as $n \rightarrow \infty$, for some $\gamma>0$ and a slowly varying function $L(\cdot)$. Then

$$
\sum_{k=0}^{\infty} p_{k} q_{n}^{* k} \sim n^{-\gamma} \sum_{k=1}^{\infty} k p_{k} L(n) \text { as } n \rightarrow \infty .
$$

Proof. By Lemma 5,

$$
\lim _{n \rightarrow \infty} \frac{q_{n}^{* k}}{q_{n}}=k, \quad k=0,1, \ldots
$$

According to Lemma 6, there exists a $C$ such that, for $k=0,1, \ldots$,

$$
\frac{q_{n}^{* k}}{q_{n}} \leq C(1+\varepsilon)^{k}, \quad n=0,1, \ldots
$$

By the Lebesgue dominated convergence theorem, we obtain

$$
\lim _{n \rightarrow \infty} \frac{\sum_{k=0}^{\infty} p_{k} q_{n}^{* k}}{q_{n}}=\sum_{k=0}^{\infty} k p_{k},
$$

which completes the proof.

Lemma 8. Suppose that a sequence $\left\{g_{n}: n=0,1, \ldots\right\}$ satisfies

$$
g_{n} \sim n^{-\gamma} L(n) \text { as } n \rightarrow \infty,
$$

where $\gamma>1$ and $L(\cdot)$ is a slowly varying function. Then

$$
\sum_{i=n}^{\infty} g_{i} \sim n^{1-\gamma} \frac{1}{\gamma-1} L(n) \text { as } n \rightarrow \infty .
$$

Proof. See Theorem 1.5.11 of [2].

Now we are ready to prove Theorem 5.

Proof of Theorem 5. (i) From (3), we have

$$
\begin{aligned}
\Pi(z) & =\frac{1-\rho}{b_{0}+(\delta-z)\left(\bar{a}_{1}-A^{+}(z)\right) /(1-z)} \\
& =\frac{1-\rho}{b_{0}+(\delta-z) \sum_{n=0}^{\infty} \bar{a}_{n+1} z^{n}} \\
& =\frac{1-\rho}{1-\rho \sum_{n=0}^{\infty}\left(\bar{a}_{n}-\delta \bar{a}_{n+1}\right) z^{n} / \rho} .
\end{aligned}
$$


Hence,

$$
\pi_{n}=\sum_{k=0}^{\infty}(1-\rho) \rho^{k} q_{n}^{* k}, \quad n=0,1, \ldots,
$$

where

$$
q_{n}=\frac{\bar{a}_{n}-\delta \bar{a}_{n+1}}{\rho}, \quad n=0,1, \ldots
$$

By Lemma 4,

$$
q_{n} \sim \frac{1-\delta}{\rho} \sum_{k=1}^{\infty}(1-\delta) \delta^{k-1} k^{\beta+1} n^{-\beta-1} L(n) \quad \text { as } n \rightarrow \infty .
$$

From Lemma 7, we obtain (25). In addition, (23) is obtained from Lemma 8.

(ii) The result follows from part (i) of this theorem and (9).

Remark 3. We have shown that the stationary distributions of the queue size and the waiting time in the DAR(1)/D/1 queue have asymptotically geometric tails if the stationary distribution of the DAR(1) process has finite support, and have regularly varying tails if the stationary distribution of the DAR(1) process has a regularly varying tail. We now have a natural question: how heavy are the tails of the queue size and the waiting time when the stationary distribution of the DAR(1) process has a moderate tail, such as a geometric tail? This requires further study.

\section{Acknowledgements}

The authors wish to thank the anonymous referee for valuable comments.

This research was supported by the Ministry of Information and Communication, Korea, under the Information Technology Research Center support program supervised by the Institute of Information Technology Assessment.

\section{References}

[1] Addie, R. G. and Zukerman, M. (1994). Performance evaluation of a single server autoregressive queue. Austral. Telecommun. Res. 28, 25-32.

[2] Bingham, N. H., Goldie, C. M. And Teugels, J. L. (1987). Regular Variation. Cambridge University Press.

[3] Choe, J. and Shroff, N. B. (2000). Use of the supremum distribution of Gaussian processes in queueing analysis with long-range dependence and self-similarity. Stoch. Models 16, 209-231.

[4] Choi, B. D., Kim, B., Hwang, G. U. And KIM, J.-K. (2004). The analysis of a multi server queue fed by discrete time autoregressive process of order 1. Operat. Res. Lett. 32, 85-93.

[5] Consul, P. C. And JaIn, G. C. (1973). A generalisation of Poisson distribution. Technometrics 15, 791-799.

[6] Cox, D. R. (1955). Some statistical methods connected with series of events. J. R. Statist. Soc. B 17, $129-164$.

[7] Cox, D. R. ANd Lewis, P. A. W. (1966). Statistical Analysis of Series of Events. Chapman and Hall, London.

[8] Drezner, Z. and Farnum, N. (1994). A correlated Poisson distribution for correlated events. Commun. Statist. Theory Meth. 23, 841-857.

[9] Elwalid, A. et al. (1995). Fundamental bounds and approximations for ATM multiplexers with applications to video teleconferencing. IEEE J. Sel. Areas Commun. 13, 1004-1016.

[10] FINCH, P. D. (1963). The single server queueing system with non-recurrent input-process and Erlang service time. J. Austral. Math. Soc. 3, 220-236.

[11] Finch, P. D. And Pearce, C. (1965). A second look at a queueing system with moving average input process. J. Austral. Math. Soc. 5, 100-106.

[12] Franken, P., König, D., Arndt, U. And Schmidt, V. (1982). Queues and Point Processes. Springer, Berlin.

[13] Gaver, D. P. AND Lewis, P. A. W. (1980). First-order autoregressive gamma sequences and point processes. Adv. Appl. Prob. 12, 727-745. 
[14] He, J. And Sohraby, K. (2001). A new analysis framework for discrete time queueing systems with general stochastic sources. In Proc. INFOCOM 2001 (Anchorage, AK, April 2001), pp. 1075-1084.

[15] Hwang, G. U. And Sohraby, K. (2003). On the exact analysis of a discrete-time queueing system with autoregressive inputs. Queueing Systems 43, 29-41.

[16] Hwang, G. U., Choi, B. D. And KIM, J.-K. (2002). The waiting time analysis of a discrete time queue with arrivals as an autoregressive process of order 1. J. Appl. Prob. 39, 619-629.

[17] JACOB, P. A. AND Lewis, P. A. W. (1983). Time series generated by mixtures. J. Time Series Anal. 4, 19-36.

[18] Kim, B., Chang, Y., KIM, Y. C. AND CHOI, B. D. (2006). A queueing system with discrete autoregressive arrivals. To appear in Performance Eval.

[19] Lawrance, A. J. And Lewis, P. A. W. (1977). An exponential moving-average sequence and point process (EMA1). J. Appl. Prob. 14, 98-113.

[20] Srinivasan, S. K. (1974). Stochastic Point Processes and Their Applications. Griffin, London.

[21] Szekli, R., Disney, R. L. And Hur, S. (1994). MR/GI/1 queues with positively correlated arrival stream. $J$. Appl. Prob. 31, 497-514.

[22] Tin, P. (1985). A queueing system with Markov-dependent arrivals. J. Appl. Prob. 22, 668-677.

[23] WolfF, R. W. (1989). Stochastic Modeling and the Theory of Queues. Prentice Hall, Englewood Cliffs, NJ. 